\title{
Numerical Study of 2-D Natural Convection in a Square Porous Cavity: Effect of Three Mode Heating
}

\author{
Giovani Elie Baptista Malomar, Cheikh Mbow, Papa Douta Tall, Abdoulaye Gueye, \\ Vieux Boukhaly Traore, Aboubaker Chedikh Beye
}

\begin{abstract}
Department of Physics, Faculty of Science and Technology, University Cheikh Anta Diop, Dakar, Senegal Email:giovanieliemalomar@yahoo.fr,mbowy@yahoo.com,papadouta@yahoo.fr,gueye812003@yahoo.fr, vieuxboukhalytraore@yahoo.fr, acbeye@refer.sn
\end{abstract}

How to cite this paper: Malomar, G.E.B., Mbow, C., Tall, P.D., Gueye, A., Traore, V.B. and Beye, A.C. (2017) Numerical Study of 2-D Natural Convection in a Square Porous Cavity: Effect of Three Mode Heating. Open Journal of Fluid Dynamics, 7, 89-104. https://doi.org/10.4236/ojfd.2017.71007

Received: January 13, 2017

Accepted: March 25, 2017

Published: March 28, 2017

Copyright $\odot 2017$ by authors and Scientific Research Publishing Inc. This work is licensed under the Creative Commons Attribution International License (CC BY 4.0).

http://creativecommons.org/licenses/by/4.0/

\begin{abstract}
The work we present in this paper is a continuation of a series of studies on the numerical study of natural convection in a square porous cavity saturated by a Newtonian fluid. The left vertical wall is subjected to a temperature varying sinusoidally in time while the right vertical wall is either at a constant temperature, or varying sinusoidally in time. The upper and lower horizontal walls are thermally adiabatic. Darcy model is used, it is also assumed the fluid studied is incompressible and obeys the Boussinesq approximation. The focus is on the effect of the modulation frequency $(10 \leq \omega \leq 100)$ on the structure of the flow and transfer thermal. The results show that the extremal stream functions $\left(\psi_{\max }\right.$ et $\left.\psi_{\min }\right)$, the average Nusselt number at the hot $\left(T_{h}\right)$ and cold $\left(T_{c}\right)$ walls respectively $\overline{N u h}$ and $\overline{N u C}$ are periodic in the range of parameters considered in this study. In comparison with the constant heating conditions, it is found that the variable heating causes the appearance of secondary flow, whose amplification depends on the frequency of modulation of the imposed temperature but also of the heating mode. The results are shown in terms of streamlines and isotherms during a flow cycle.
\end{abstract}

\section{Keywords}

Natural Convection, Sinusoidal Temperature, Porous Media, Numerical Study

\section{Introduction}

The natural convection in confined porous media saturated by fluid is fundamental in the fields of engineering and physics. This interest arises from the importance of this heat transfer mode in various engineering fields such as storage 
of the thermal energy, solar energy collectors, thermal design for the buildings, cooling of electronic components (Alves and Altemani [1], Kuznetsov and Sheremet [2], Sheremet and Pop [3]), the underground spread of pollutants (Bagchi and Kulacki [4]). The literature on the convective flow in porous media is abundant. An excellent review of most of these studies is in the books (Nield and Bejan [5], Pop and Ingham. [6], Vafai [7], Ingham and Pop [8]) that give a complete overview of the current state of research in this area. However, most of these studies, theorical and experimental, on side convection, consider boundary conditions thermal constant (temperature or flux constant heat). Excellent review articles including one written by Baytas and Pop [9], give detailed accounts results obtained with these boundaries conditions. However, these boundary conditions do not reflect what is encountered in many practical situations where the temperature gradient is a function of time. This is the case of electronic components that dissipate power intermittently during operation on/of. Mastering the gradient behavior heat in these real situations can be used to control the flow convective. For example, it may be used to control the quality and structure of a solid resulting from solidification of an alloy by influencing the process of transport. The effect of the modulation of temperature on natural convection in cavity fluid medium was several time studied. For example, Schaladow et al. [10] studied the case of a cavity subjected to a temperature which increases linearly each time. Both considered simulations (numerical and experimental) show that the flow and temperature field are very little affected by these boundary conditions. For their part, Kazmierczak and Chinoda [11] have numerically studied the effect of temperature varying sinusoidally in time on fluid flow and heat transfer in a square cavity. These authors showed that the heat transfer means in time is substantially insensitive to the periodic change of the wall temperature. Lage and Bejan [12], studied enclosures with one sidewall heated using a pulsating heat flux and the other sidewall cooled at constant temperature. They showed that at high Rayleigh numbers, the buoyancy-driven flow has the tendency to resonate to the periodic heating that has been supplied from the side. Lage et al. [13], have studied the phenomenon of flow interference caused by discrete, solid objects placed inside fluid saturated enclosures under natural convection. Their analysis confirms and quantifies the predominance of the horizontal interference when the enclosure becomes shallow $(A>1)$. Seyf and Rassoulinejad-Mousavi [14] proposed a new analytical solution for 2D Darcy-Brinkman equations in porous channels filled with porous media subjected to various boundary conditions at walls. The obtained ODE is solved analytically using homotopy perturbation method. It was shown that there is an excellent agreement between the presented models and the results of the CFD and previous works. Rassoulinejad-Mousavi and Yaghoobi [15] studied the effect of form drag term on the viscous dissipation through a parallel plate channel packed with a porous medium with isoflux or isothermal condition at the walls. Abourida et al. [16], have examined the effect of imposed sinusoidal temperatures and the thermophysical ones on the fluid flow and heat transfer within the cavity. They 
showed that all the obtained solutions concerning the fluid flow are periodic, with a period identical to that imposed to the variable temperatures. Note that many of these works consider cavities in which the temperature of cold wall is maintained constant. However, very little work is done on natural convection in a cavity filled with a porous medium with boundary conditions thermal periodic in time and using the Darcy model. Some of the documents dealing with this problem are, Saeid [17], Malomar et al. [18]. The main objective of the present study is to contribute to the enrichment of the kind of problem by examining the effect of the imposed sinusoidal temperatures parameter (frequency) on the structure of the flow and transfer thermal. We study numerically natural convection unsteady in a square cavity, filled with a porous medium and the vertical walls are subjected at least to a temperature varying sinusoidally with time. The horizontal walls are thermally adiabatic and Darcy model was used.

\section{Materials and Methods}

\subsection{Basic Equations}

A schematic geometry of the problem is shown in Figure 1, where $x^{\prime}$ and $y^{\prime}$ are the cartesian coordinates and $H^{\prime}$ is the size of the walls. This is a square porous cavity two-dimensional and the horizontal walls are assumed to be thermally adiabatic. All walls of the cavity are assumed to be impermeable. At the same, the vertical walls, left wall (hot $T_{h}$ ) and right wall (cold $T_{c}$ ) are subjected at least to a temperature varying sinusoidally with time such that:

$$
\begin{gathered}
T_{h}^{\prime}=T_{R}+\Delta T^{\prime}+a^{\prime} \sin \left(\omega^{\prime} t^{\prime}\right) \\
T_{c}^{\prime}=T_{R}+a^{\prime} \sin \left(\omega^{\prime} t^{\prime}+\phi^{\prime}\right)
\end{gathered}
$$

where $T_{R}$ is the reference temperature (in our study $T_{R}=\bar{T}_{c}^{\prime}$ ), $\Delta T^{\prime}=\bar{T}_{h}^{\prime}-\bar{T}_{c}^{\prime}$ is the average temperature difference, $a^{\prime}$ represents the amplitude of the

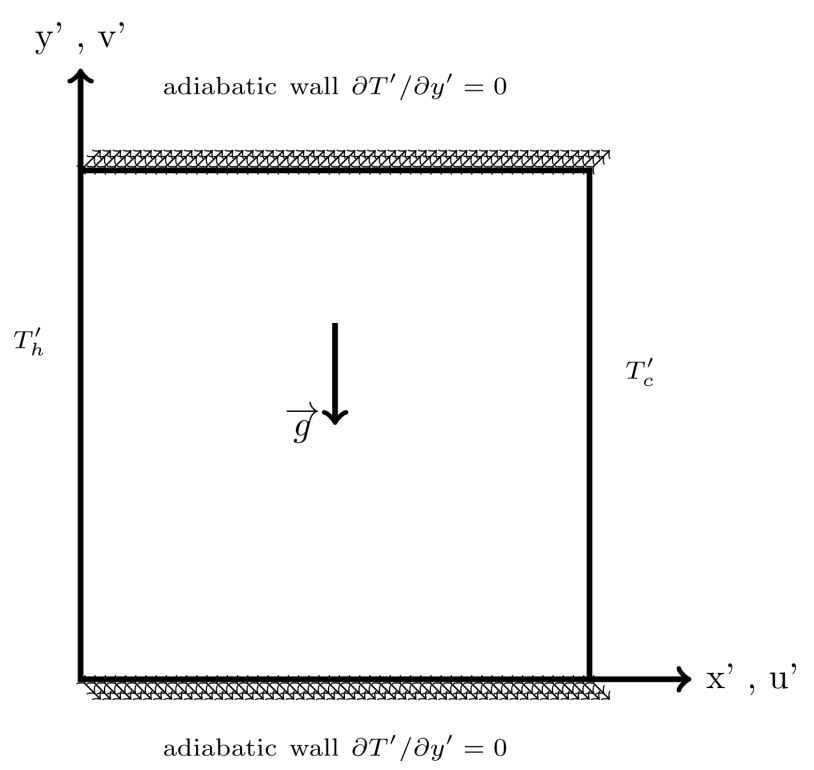

Figure 1. Schematic diagram of the physical model and coordinate system. 
modulation, $\phi^{\prime}$ the phase angle and $\omega^{\prime}$ the modulation frequency. The following three cases are considered: 1) only the left wall's temperature is modulated, the right wall is held at constant temperature; 2) the temperatures modulation are out-of-phase, i.e. $\left.\phi^{\prime}=\pi ; 3\right)$ the temperatures modulation are in-phase, i.e. $\phi^{\prime}=0$. The Darcy-Boussinesq approximation is employed. Isotropy, homogeneity and local thermal equilibrium in the porous medium are assumed. Under these assumptions, the equations governing the problem is the equation continuity, the equation of motion and the energy equation, respectively (see Nield and Bejan [5]):

$$
\begin{gathered}
\frac{\partial u^{\prime}}{\partial x^{\prime}}+\frac{\partial v^{\prime}}{\partial y^{\prime}}=0 \\
\frac{\partial v^{\prime}}{\partial x^{\prime}}-\frac{\partial u^{\prime}}{\partial y^{\prime}}=\frac{k g \rho_{0} \beta_{T}}{\mu} \frac{\partial T^{\prime}}{\partial x^{\prime}} \\
\sigma \frac{\partial T^{\prime}}{\partial t^{\prime}}+u^{\prime} \frac{\partial T^{\prime}}{\partial x^{\prime}}+v^{\prime} \frac{\partial T^{\prime}}{\partial y^{\prime}}=\alpha \nabla^{2} T^{\prime}
\end{gathered}
$$

where $u^{\prime}$ and $v^{\prime}$ are the velocity components along $x^{\prime}$ and $y^{\prime}, T^{\prime}$ is the fluid temperature, $t^{\prime}$ is the time, $\beta_{T}=-1 / \rho_{0}\left(\partial \rho / \partial T^{\prime}\right)_{p}$ is the coefficient of thermal expansion. The quantities $\sigma$ and $\alpha$ are defined by $\alpha=k_{m} /(\rho c)_{f}$ and $\sigma=(\rho c)_{m} /(\rho c)_{f}$ where $k_{m}$ is the thermal conductivity (solid phase + fluid phase).

Equations (3)-(5) are subject to the following boundary conditions and initial:

$$
\begin{cases}u^{\prime}\left(x^{\prime}, y^{\prime}, 0\right)=v^{\prime}\left(x^{\prime}, y^{\prime}, 0\right)=0 ; & T^{\prime}\left(x^{\prime}, y^{\prime}, 0\right)=0 \\ u^{\prime}\left(0, y^{\prime}, t^{\prime}\right)=0 ; & T^{\prime}\left(0, y^{\prime}, t^{\prime}\right)=T_{h}^{\prime} \\ u^{\prime}\left(L^{\prime}, y^{\prime}, t^{\prime}\right)=0 ; & T^{\prime}\left(L^{\prime}, y^{\prime}, t^{\prime}\right)=T_{c}^{\prime} \\ v^{\prime}\left(x^{\prime}, 0, t^{\prime}\right)=0 ; & \partial T^{\prime}\left(x^{\prime}, 0, t^{\prime}\right) / \partial y^{\prime}=0 \\ v^{\prime}\left(x^{\prime}, L^{\prime}, t^{\prime}\right)=0 ; & \partial T^{\prime}\left(x^{\prime}, L^{\prime}, t^{\prime}\right) / \partial y^{\prime}=0\end{cases}
$$

One can introduce a stream function $\psi^{\prime}$ defined by $u^{\prime}=\partial \psi^{\prime} / \partial y^{\prime}$ and $v^{\prime}=-\partial \psi^{\prime} / \partial x^{\prime}$ so that Equation (3) is satisfied. Then the governing Equations (3)-(5) can be rewritten with the following dimensionless variables:

$$
\begin{aligned}
& (x, y)=\left(\frac{x^{\prime}}{H^{\prime}}, \frac{y^{\prime}}{H^{\prime}}\right) ; \quad(u, v)=\left(\frac{u^{\prime} H^{\prime}}{\alpha}, \frac{v^{\prime} H^{\prime}}{\alpha}\right) ; \quad t=\frac{t^{\prime} \alpha}{\sigma H^{\prime 2}} \\
& T=\frac{T^{\prime}-\bar{T}_{c}^{\prime}}{\bar{T}_{h}^{\prime}-\bar{T}_{c}^{\prime}} ; \quad \omega=\frac{\omega^{\prime}}{2 \pi} \frac{\sigma H^{\prime 2}}{\alpha} ; \quad \psi=\frac{\psi^{\prime}}{\alpha} ; \quad a=\frac{a^{\prime}}{\bar{T}_{h}^{\prime}-\bar{T}_{c}^{\prime}}
\end{aligned}
$$

$\tau^{\prime}$ represents the dimensional period and is connected to the modulation frequency dimensional by the following equation $\omega^{\prime}=2 \pi / \tau^{\prime}$.

Substituting (7) in (3)-(5) we obtain the following dimensionless governing equations:

$$
\begin{gathered}
\frac{\partial^{2} \psi}{\partial x^{2}}+\frac{\partial^{2} \psi}{\partial y^{2}}=-R_{a} \frac{\partial T}{\partial x} \\
\frac{\partial T}{\partial t}+\frac{\partial \psi}{\partial y} \frac{\partial T}{\partial x}-\frac{\partial \psi}{\partial x} \frac{\partial T}{\partial y}=\frac{\partial^{2} T}{\partial x^{2}}+\frac{\partial^{2} T}{\partial y^{2}}
\end{gathered}
$$


associated with initial and boundary conditions

$$
\begin{cases}\psi(x, y, 0)=0 ; & T(x, y, 0)=0 \\ \psi(0, y, t)=0 ; & T(0, y, t)=T_{h} \\ \psi(1, y, t)=0 ; & T(1, y, t)=T_{c} \\ \psi(x, 0, t)=0 ; & \partial T(x, 0, t) / \partial y=0 \\ \psi(x, 1, t)=0 ; & \partial T(x, 1, t) / \partial y=0\end{cases}
$$

where $T_{h}=1+a \sin (\omega t)$ et $T_{c}=a \sin (\omega t+\phi)$ and $R_{a}=k \rho_{0} g \beta_{T} H^{\prime} \Delta T^{\prime} / \mu \alpha$ represents the Rayleigh number.

At each time $t$ the average Nusselt numbers at the vertical walls (hot and cold) are defined by, respectively:

$$
\overline{N u h}=-\int_{0}^{1}\left(\frac{\partial T}{\partial x}\right)_{x=0} \mathrm{~d} y \quad ; \quad \overline{N u c}=-\int_{0}^{1}\left(\frac{\partial T}{\partial x}\right)_{x=1} \mathrm{~d} y
$$

\subsection{Numerical Method}

The equations of motion (8) and energy (9) associated with the boundary conditions (10) are discretized by a finite difference scheme, centred and accurate to the second order. The energy equation is then solved by the implicit method of alternating directions (ADI). The linear discretized equations were solved by Thomas algorithm. For equation of motion, the obtained linear discretized equation was solved by the sucessive over-relaxation method. Uniform grids have been selected in both the $x$ and $y$ direction. We have developed a numerical code with Fortran 95.

The calculation stops when between two time steps, the following condition is satisfied by the stream function:

$$
\sum_{i, j}\left(\left|\frac{\psi_{i, j}^{n+1}-\psi_{i, j}^{n}}{\psi_{i, j}^{n+1}}\right|\right) \leq 10^{-5}
$$

Preliminary tests on the influence of the mesh have allowed us to retain a uniform mesh size of $120^{*} 120$. The time step used is $10^{-4}$. The present numerical code have been validated against the works of Walker and Homsy [19], Bejan [20], Beckerman et al. [21], Moya et al. [22]; Manole and Lage [23], Baytas and Pop [9] for the steady state natural convection in a square porous cavity with isothermal vertical and adiabatic horizontal walls. Table 1 shows the values of the average Nusselt number computed for various Rayleigh numbers in the range $10-10^{3}$ in comparison with other authors.

Note that all numerical simulations are initialized by considering a conductive state and constant heating conditions. When steady regime is established, we introduce the excitatory temperatures and expecte the establishment of a periodic regime.

\section{Results and Discussion}

\subsection{Influence of the Modulation Frequency}

To highlight the effect of $\omega$ frequency modulation, we present the temporal 
evolution of the functions $\psi_{\max }, \psi_{\min }, \overline{N u C}$ and $\overline{N u h}$ for the three types of oscillatory heating under the following conditions $a=0.8 ; R a=10^{3}$ and $10 \leq \omega \leq 100$. Note that Figure 2(a), Figure 3(a) and Figure 4(a) show the temporal evolutions of $T_{h}$ and $T_{c}$ for $\omega=30$.

Thus Figures 2(b)-(e) respectively show the temporal evolution of the

Table 1. Comparison of the average Nusselt number of the hot wall.

\begin{tabular}{cccc}
\hline & \multicolumn{3}{c}{$\overline{N u h}$} \\
Paper & $R a=10$ & $R a=100$ & $R a=1000$ \\
\hline Walker and Homsy [19] & - & 3.10 & 12.96 \\
Bejan [20] & - & 4.20 & 15.80 \\
Beckerman et al. [21] & - & 3.11 & - \\
Moya et al. [22] & 1.065 & 2.80 & 13.64 \\
Manole and Lage [23] & - & 3.12 & 14.06 \\
Baytas and Pop [9] & 1.079 & 3.16 & 15.60
\end{tabular}
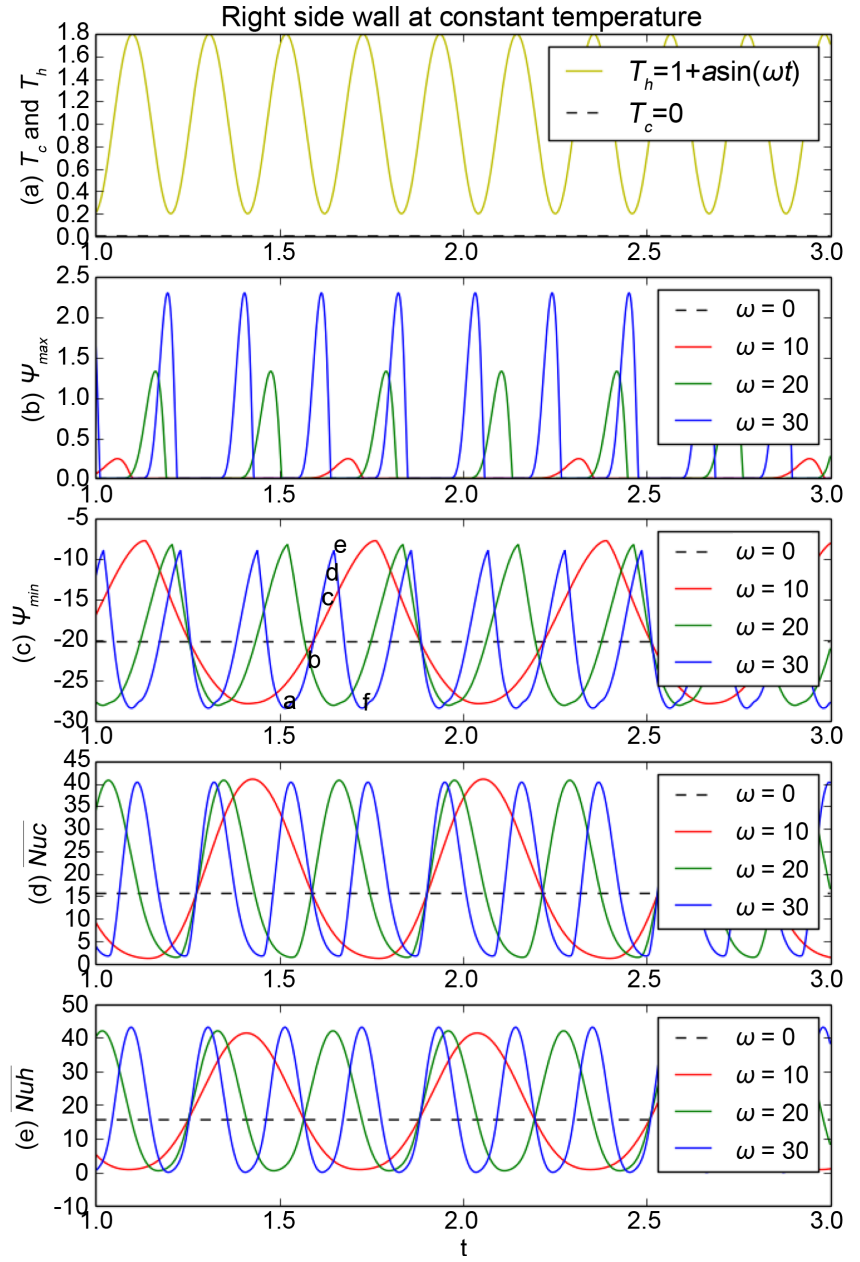

Figure 2. Effect of $\omega$ for $a=0.8$ and $R a=10^{3}$ on (from top to bottom): (a) $T_{h}(t)$ and $T_{c}(t)$, (b) $\psi_{\max }(t),(\mathrm{c}) \psi_{\min }(t)$, (d) $\overline{N u c}(t)$ and (e) $\overline{N u h}(t)$. 


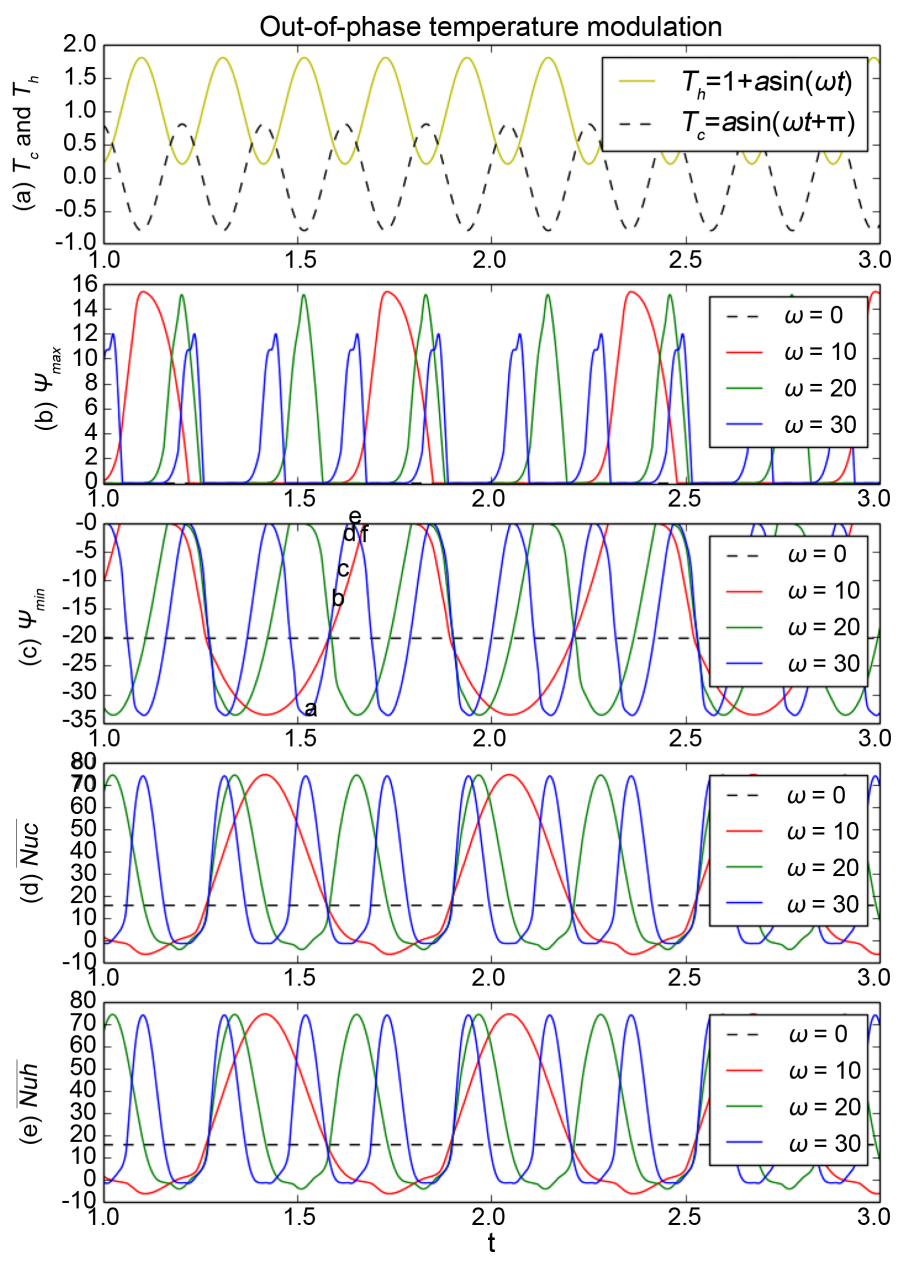

Figure 3. Effect of $\omega$ for $a=0.8$ and $R a=10^{3}$ on (from top to bottom): (a) $T_{h}(t)$ and $T_{c}(t)$, (b) $\psi_{\text {max }}(t)$, (c) $\psi_{\text {min }}(t)$, (d) $\overline{N u c}(t)$ and (e) $\overline{N u h}(t)$.

functions $\psi_{\max }, \psi_{\min }, \overline{N u C}$ and $\overline{N u h}$ in the case where only the temperature of the hot wall $\left(T_{h}\right)$ is modulated. The curves of Figures $2(\mathrm{~b})-(\mathrm{e})$ show that all the solutions obtained are periodic, of periods equal to those of the excitation imposed temperature. In the frequency range $10 \leq \omega \leq 30$, the amplitudes of functions $\psi_{\min }, \overline{N u C}$ and $\overline{N u h}$ remain very close while those of $\psi_{\max }$ increases when $\omega$ increases. We also note that the nature of sinusoidal oscillations is kept in $\overline{N u h}$. Figure 2(e) shows that for $\omega=30, \overline{N u h}$ takes negative values during a brief portion of the cycle.

In the case where the temperatures excitatory evolve in phase opposition, Figures 3(b)-(e) show that all the solutions obtained are periodic, periods equal to those the imposed excitatory temperatures. In the frequency range $10 \leq \omega \leq 30$, the amplitudes of the functions presented remain very close except for $\psi_{\text {max }}(t)$ where there is a notable decrease in the amplitude when $\omega$ go of 20 to 30. For this heating mode is also observed better competition between cells rotating in clockwise direction and cells rotating in counterclockwise direction, resulting in a cancellation of $\psi_{\min }$ during brief part of the cycle. During this time interval, the flow is monocellular, characterized by a presence of cell rotating in a clockwise 


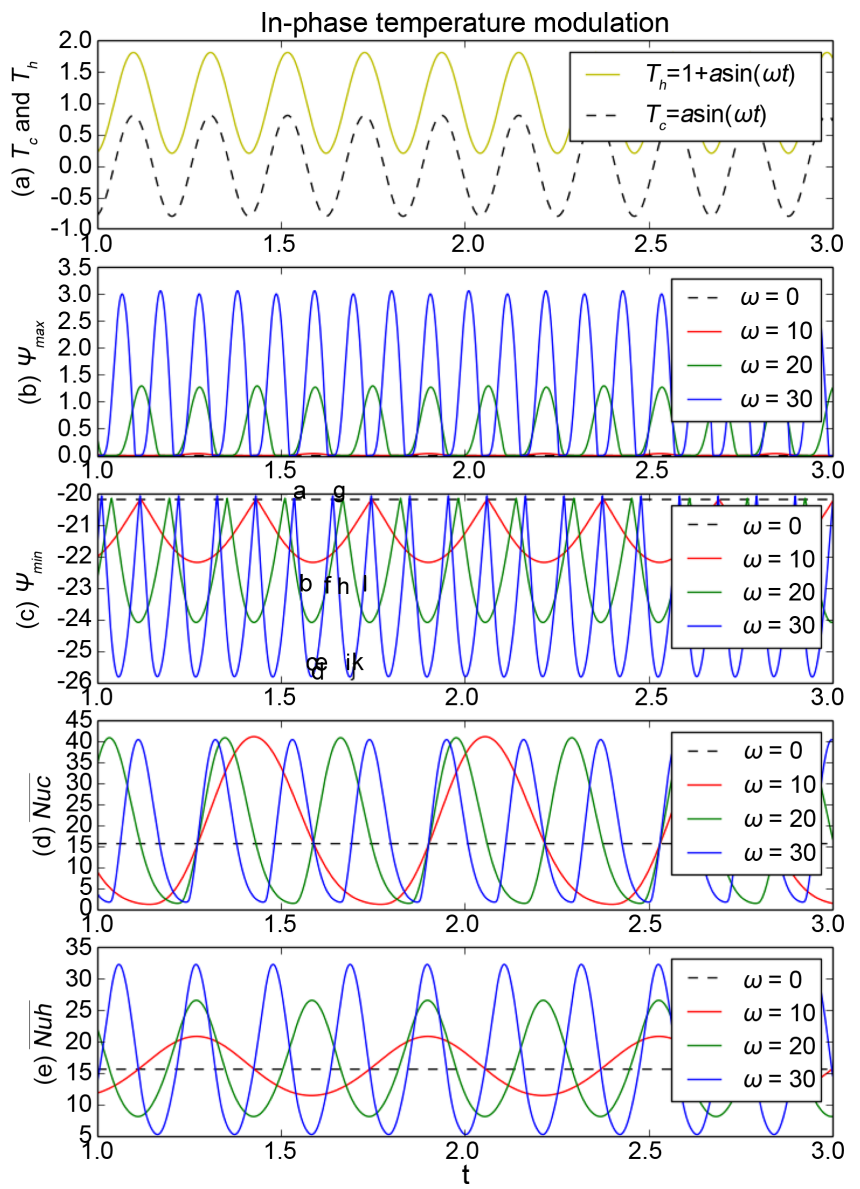

Figure 4. Effect of $\omega$ for $a=0.8$ and $R a=10^{3}$ on (from top to bottom): (a) $T_{h}(t)$ and $T_{c}(t),(\mathrm{b}) \psi_{\max }(t),(\mathrm{c}) \psi_{\min }(t)$, (d) $\overline{N u c}(t)$ and (e) $\overline{N u h}(t)$.

direction and of which intensity decreases when $\psi_{\min }$ tends to 0 . Unlike the case where only the temperature of the hot wall is variable, the sinusoidal nature of the excitation is not conserved for the different functions. Figures 3(d)-(e) show that during part of the cycle, the average Nusselt numbers $\overline{N u C}$ and $\overline{N u h}$ take negative values. These negative values found, are justified by the fact that for amplitudes $(a>0.5)$ the cold wall acquires temperatures higher than the wall supposed to be hot. This then results in a transfer of heat from the cold wall inwardly of the cavity as soon as this wall becomes hotter than the surrounding fluid. Thereafter the fluid surrounding the hot wall becomes hotter than this wall it results a heat transfer from the fluid outwardly the cavity via the hot wall.

For the last mode of heating studied where excitatory temperatures are in phase, Figures 4 (b)-(e) show that only functions $\overline{N u C}$ and $\overline{N u h}$ retain the sinusoidal look and the period imposed on excitatory temperatures while $\psi_{\text {max }}$ and $\psi_{\min }$ are periodic, with a period which is half that of excitatory temperatures. These figures also show that the amplitudes of functions increase when ${ }_{o}$ mega increases except $\overline{N u C}$ where the amplitudes are very close to the range of frequencies considered. However, we note that the most important effects of frequency modulation $\omega$ are rated for high values of this parameter 
( $\omega>100$ ). But for convenience we chose to study the functions for $10 \leq \omega \leq 30$ because even for this range frequency, important differences qualitative and quantitative are observed.

\subsection{Streamlines and Isotherms}

To understand the details of flow and heat transfer in the cavity, we produced streamlines and isotherms for each of the three modes of heating, during a flow cycle for $a=0.8 ; R=10^{3}$ and $\omega=30$.

Before discussing the results for the oscillatory regime, we produced streamlines and isotherms in steady state $(a=0)$ for $R a=10^{3}$. Note that in the case of constant heating ( $a=0$ ), the flow in the cavity remains monocell, consisting of cell rotating in clockwise direction and having a symmetry relative to the center of the cavity (see Figure 5).

In the case where only the hot temperature $\left(T_{h}\right)$ is modulated, Figures 6(a)-(f) show the streamlines (left) and isotherms (right) at times corresponding to the letters a, b, c, d, e and f in Figure 2. When the temperature of the hot wall is at its maximum $\left(T_{h}=1.798\right)$ the flow field is dominated by a cell rotating in clockwise direction and occupying the entire cavity (Figure 6(a)). The maximum of the absolute value of $\psi_{\min }\left(\left|\psi_{\min }\right|_{\max }\right)$ being located in the neighboring of center-left of the cavity and compared to the constant heating case $(a=0)$, symmetry is broken. The structure of the isotherms show the existence of high thermal gradients in the vicinity of active walls, which leads to the maximum heat transfer and Nusselt numbers at this time (a). It is also observed that when $T_{h}$ decreases, $\left|\psi_{\min }\right|_{\max }$ moves and is located in the neighboring of center-right of the cavity (Figure 6(b)). The gradual reduction of $T_{h}$ is accompanied by a decrease in the intensity of this cell rotating in clockwise and promotes the appearance of a secondary cell rotating counter-clockwise and localized to the upper left corner of the cavity (Figure 6(c) and Figure 6(d)). This is explained by the existence of a quantity of fluid surrounding the hot wall and having a higher temperature than the hot wall. At the same moment the structure of
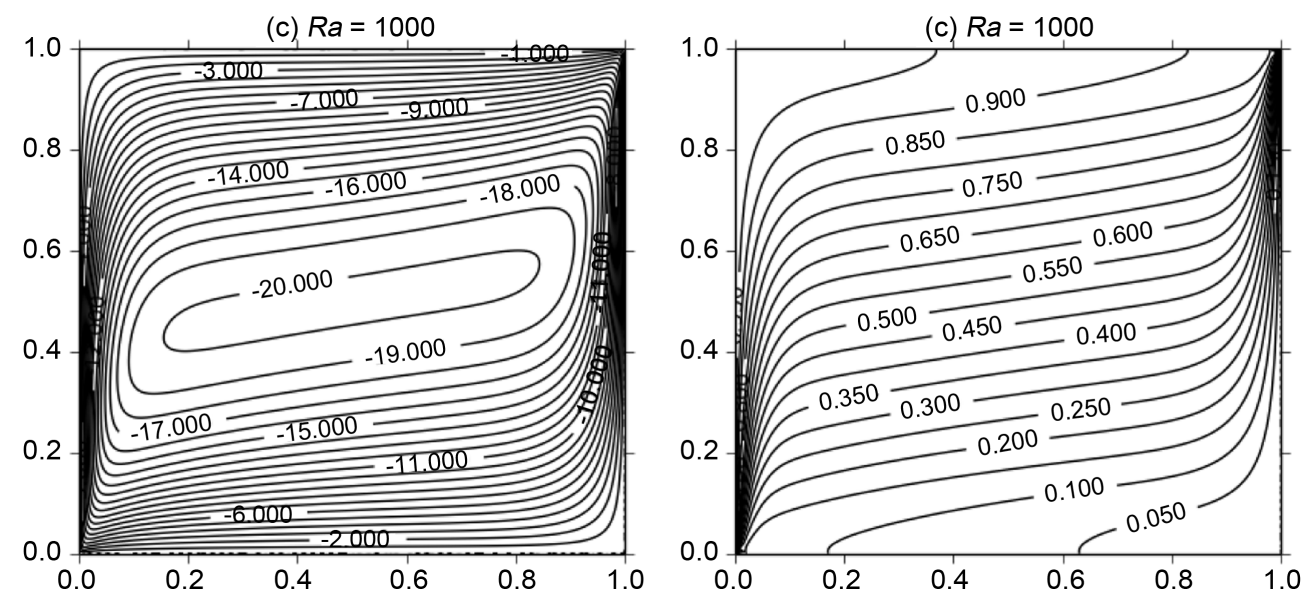

Figure 5. Streamlines (left) and isotherms (right) for $R a=10^{3}$ : Steady state solution. 

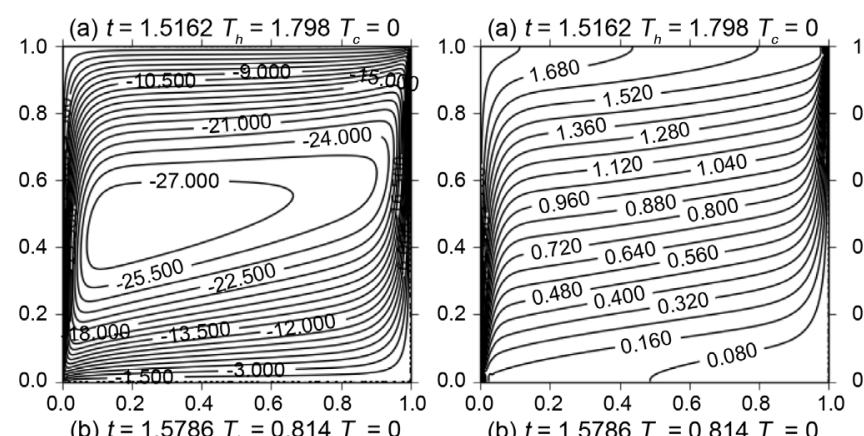

(d) $t=1.6266 T_{h}=0.204 T_{c}=0$

(d) $t=1.6266 T_{0}=0.204 T_{c}=0$
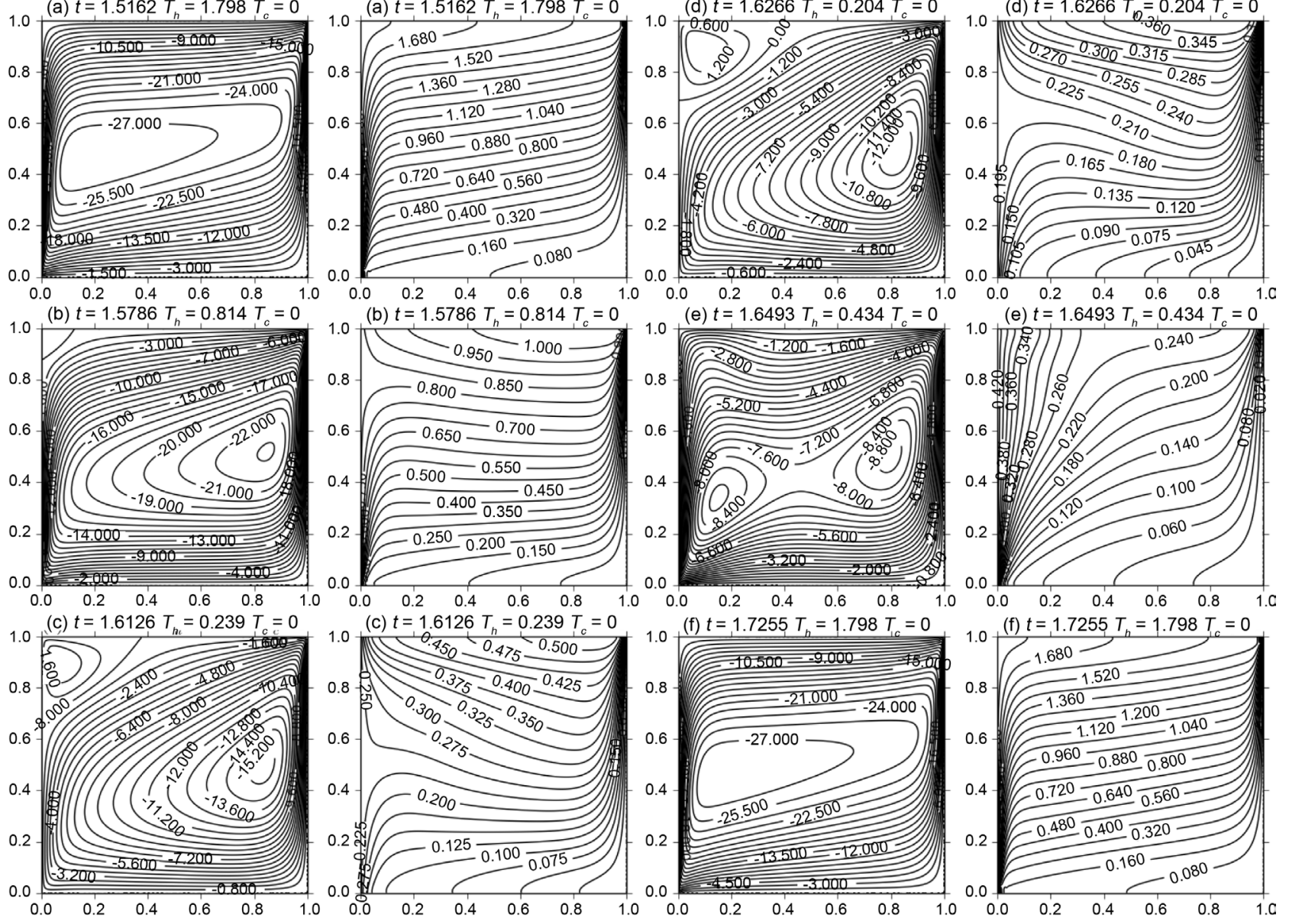

Figure 6. (a)-(f) Streamlines (left) and isotherms (right) over cycle for $R a=10^{3}, a=0.8$ and $\omega=30$.

isotherms show a spacing between isotherms at the hot wall, which results in significant reduction in heat transfer, demonstrated by the average Nusselt numbers are near their minimum values. Once that the temperature of the hot wall again believes in leaving its minimum value, the secondary cell rotating in counterclockwise direction decreases in intensity and eventually disappear in favor of the formation of a cell rotating in clockwise direction, near the hot wall and which is added to the cell rotating in clockwise direction already in the vicinity of the cold wall (Figure 6(e)). The structure of isotherms show that heat transfer are near to minimum values. Beyond the moment (e), it is gradually forms a main cell rotating in clockwise direction and occupying the entire cavity in end of the cycle (Figure 6(f)).

In the case where temperatures evolve in phase opposition, Figures 7(a)-(f) show streamlines (left) and isotherms (right) at times corresponding to the letters a, b, c, d, e and f in Figure 3. We note that at the moment (a) where the hot wall is at its maximum value, $T_{h}=1.8$ and the cold wall is at its minimum value, $T_{c}=-0.8$ the flow regime is monocell, dominated by one cell rotating in a clockwise direction and occupying the entire cavity. We observe that also at time (a) the structure of isotherms shows stratification of the temperature around the center of the cavity and of high gradients thermal around the active 

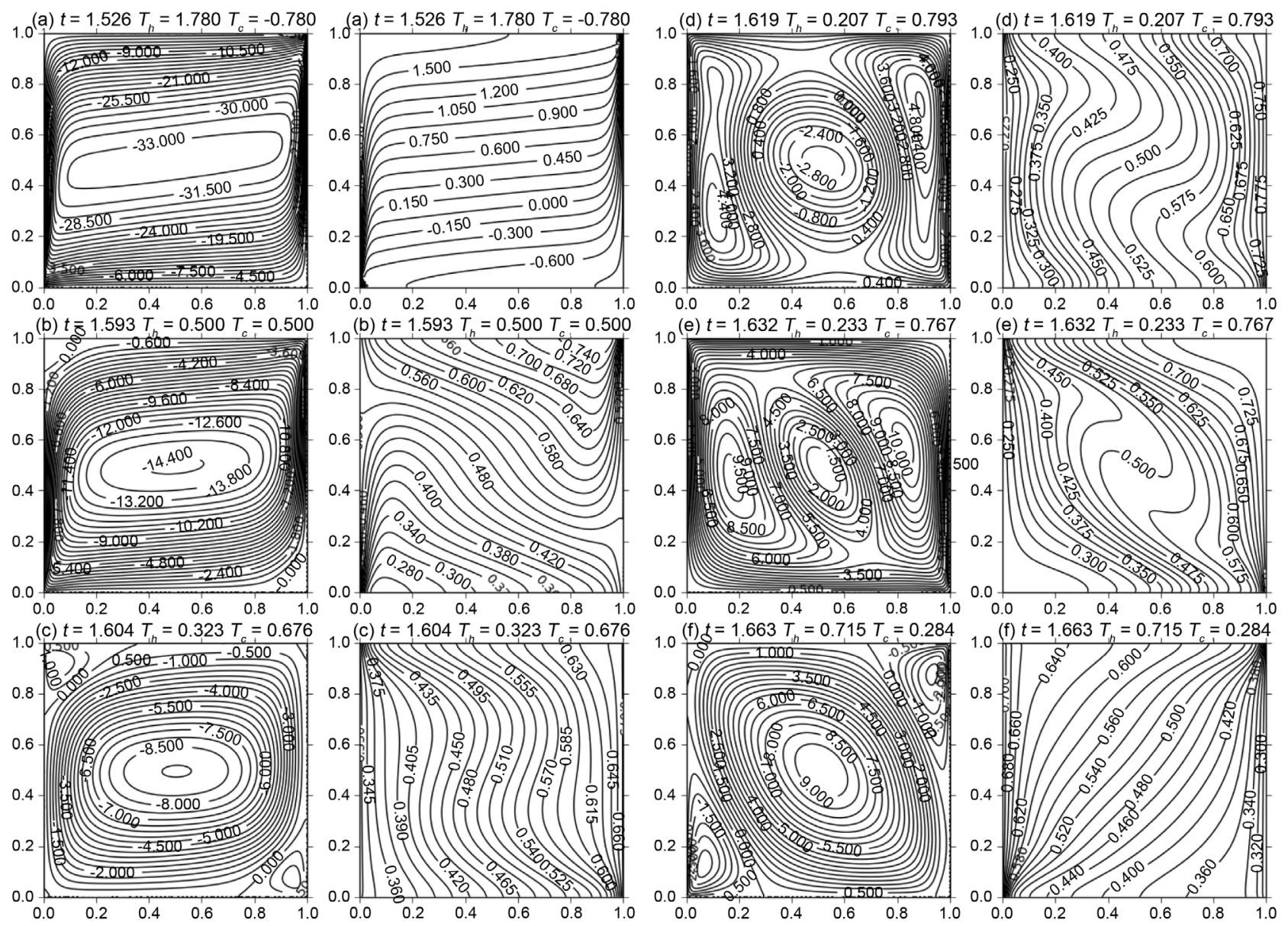

Figure 7. (a)-(f) Streamlines (left) and isotherms (right) over cycle for $R a=10^{3}, a=0.8$ and $\omega=30$.

walls (Figure 7(a)). Evolving in the cycle, $T_{h}$ decreases and $T_{c}$ increases, there is a weakening of the main cell. This weakening of the main cell is further complicated by the occurrence of two recirculation cells rotating in a counterclockwise direction, one at the upper left corner and the other at the lower right corner (Figure $7(\mathrm{~b})$ and Figure $7(\mathrm{c})$ ). The appearance of these two negative cells cause a spacing of the isotherms in the vicinity of active walls whence a heat transfer reduction. These secondary cells expands along the active walls at the expense of the cell rotating in a clockwise direction and causing a reduction in heat transfer (Figure $7(d)$ ). Continuing in the cycle, the flow will consist of three cells rotating in counterclockwise (Figure 7(e)) and thereafter for a short time, the flow is dominated by one cell rotating in a counterclockwise direction (not shown here). Between moments (d) and (e) structure of isotherms attests that heat transfer are virtually reduced to zero. In the second part of the cycle, the cell rotating in a clockwise direction will play a role similar to the one played by the cell rotating in a counterclockwise direction during the first part of the cycle. However the two recirculation cells rotating in a clockwise direction appear in the corner of level upper right and lower left (Figure $7(f)$ ). Consequently, these secondary cells rotating in a clockwise direction expands along the active walls to the detriment of the cell rotating in a counterclockwise direction 
and thereafter a cycle with a monocellular cell rotating in a clockwise direction starts again.

In case where the two temperatures are changing in phase, Figures 8(a)-(1) show the streamlines (left) and isotherms (right) corresponding to the instants a, $\mathrm{b}, \ldots$ and 1 in Figure 4. At time (a), $T_{h}$ and $T_{c}$ are around them maximum, the flow in cavity is monocellular, characterized by the presence of a cell rotating in a clockwise direction (Figure 8(a)). Evolving in the cycle, at end of first half of the flow cycle, we note with decreasing temperatures the appearance of a secondary cell rotating counterclockwise in the upper left corner of the cavity. $\left|\psi_{\min }\right|_{\max }$ is located in the center right of the cavity (Figures $8(\mathrm{~b})-(\mathrm{e})$ ). The appearance of this secondary cell is explained by the existence of a quantity of fluid surrounding the hot wall and having a higher temperature than the hot wall. Beyond the moment (e), the secondary cell is weakened (Figure $8(f)$ ) and eventually disappear at the end of flow cycle (Figure $8(\mathrm{~g})$ ) when $T_{h}$ and $T_{c}$ take minimum values. At time (a) the structure of the isotherms show relatively high thermal gradients around the active walls unlike the moments b, c, d, e and $\mathrm{f}$ where it has a spacing of the isotherms at the upper half of the hot wall, which leads the reduction of heat transfer. Figure $8(\mathrm{a})$ and Figure $8(\mathrm{~g})$ show a similar flow state qualitatively but different from the quantitative standpoint as illustrated by the temporal variations of $\overline{N u h}$ and $\overline{N u C}$. Continuing to evolve in the cycle, $T_{h}$ and $T_{c}$ grow again, we see that $\left|\psi_{\min }\right|_{\max }$ moves to the left center of the cavity, which leads to creation of a secondary cell (Figures 8(i)-(k)). However the secondary cell that forms and rotating in a counterclockwise is located in the lower right corner. His apparition is due to the existence of a quantity of fluid in the vicinity of the cold wall whose temperature is lower than the cold wall. This secondary cell is weakened (Figure 8(1)) and disappear at the end of flow cycle. We also noticed that flow conditions (Figures $8(\mathrm{~g})-(1)$ ) can be obtained from the flow conditions (Figures $8(\mathrm{a})-(\mathrm{f})$ ) respectively, by rotating the cavity in its plan of 180 degree. The structure of isotherms shows a spacing of the isotherms at the lower half of the cold wall.

\section{Conclusions}

The numerical study of unsteady natural convection in a porous cavity square whose side walls are subjected at least to a temperature varying sinusoidally with time was investigated. The mathematical model used is that of Darcy in the Boussinesq approximation. The algorithm was validated by direct comparison with previously published work and the results were considered in good agreement. Streamlines and isotherms were produced for $R a=10^{3}, a=0.8$ et $w=30$ for each of the three mode heating.

Based on the results found in this study, we remark that the oscillatory heating causes the appearance of secondary flow, whose amplification depends on the frequency of modulation of the imposed temperature but also of the heating mode. In addition the imposed oscillatory heating improves thermal transfer compared to the constant heating and constitutes the best way to remove the 

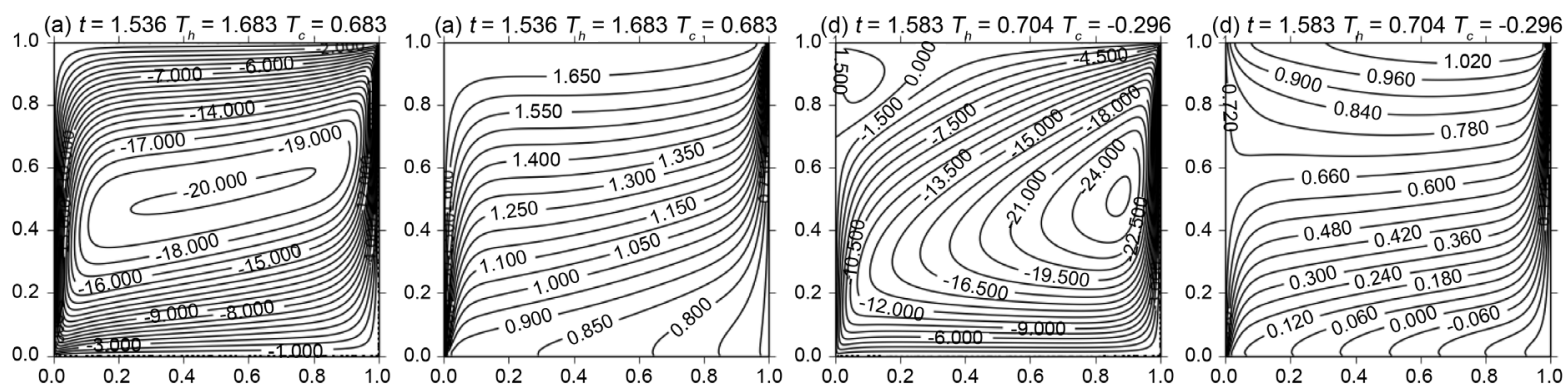

(b) $t=1.554 T_{h}=1.386 T_{c}=0.386$
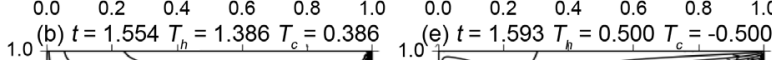

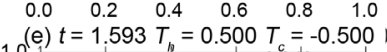
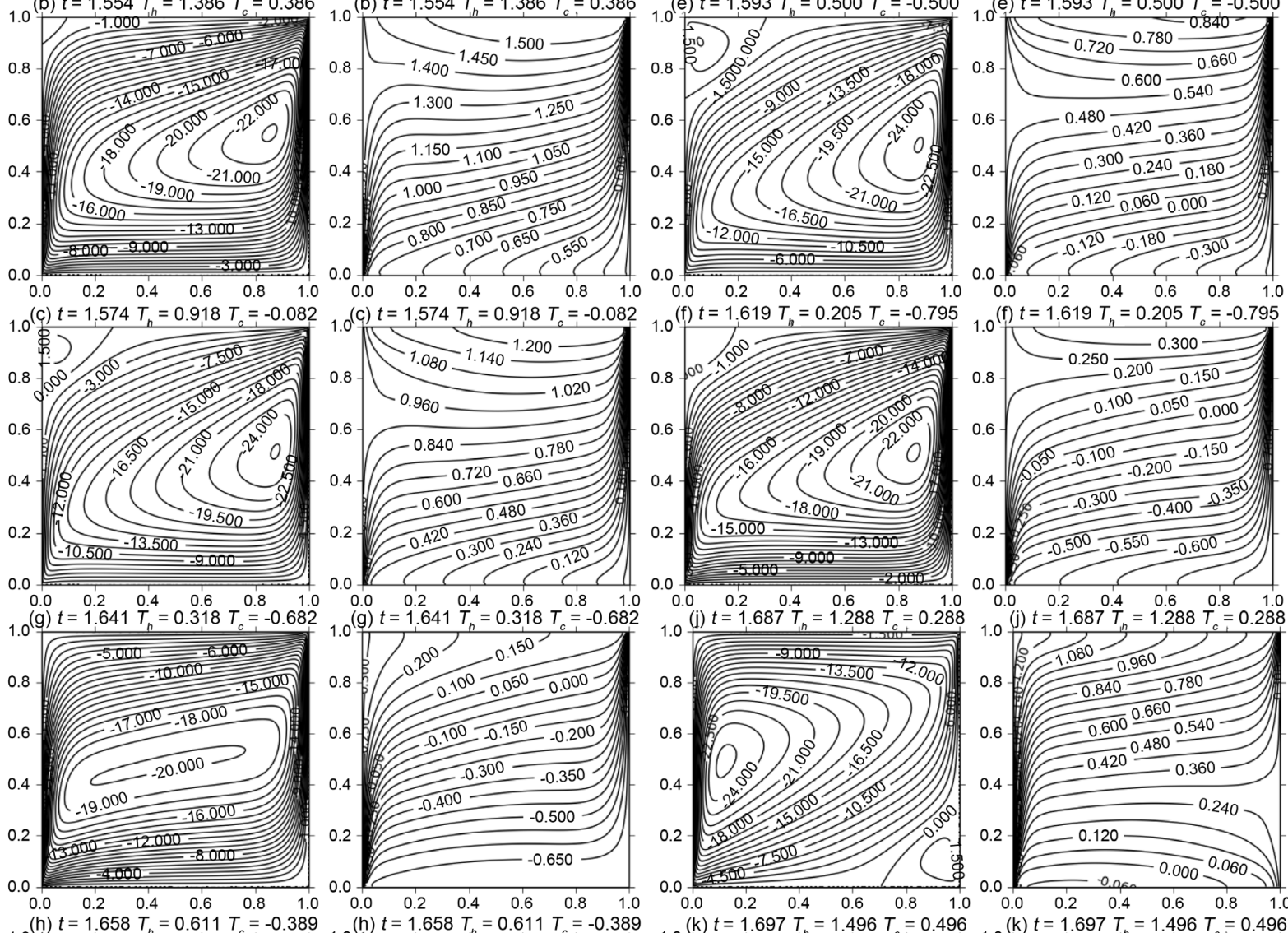

$\begin{array}{ccccc}0.0 & 0.2 & 0.4 & 0.6 & 0.8 \\ \text { (h) } t=1.658 & T=0.611 & T= & -0.389\end{array}$
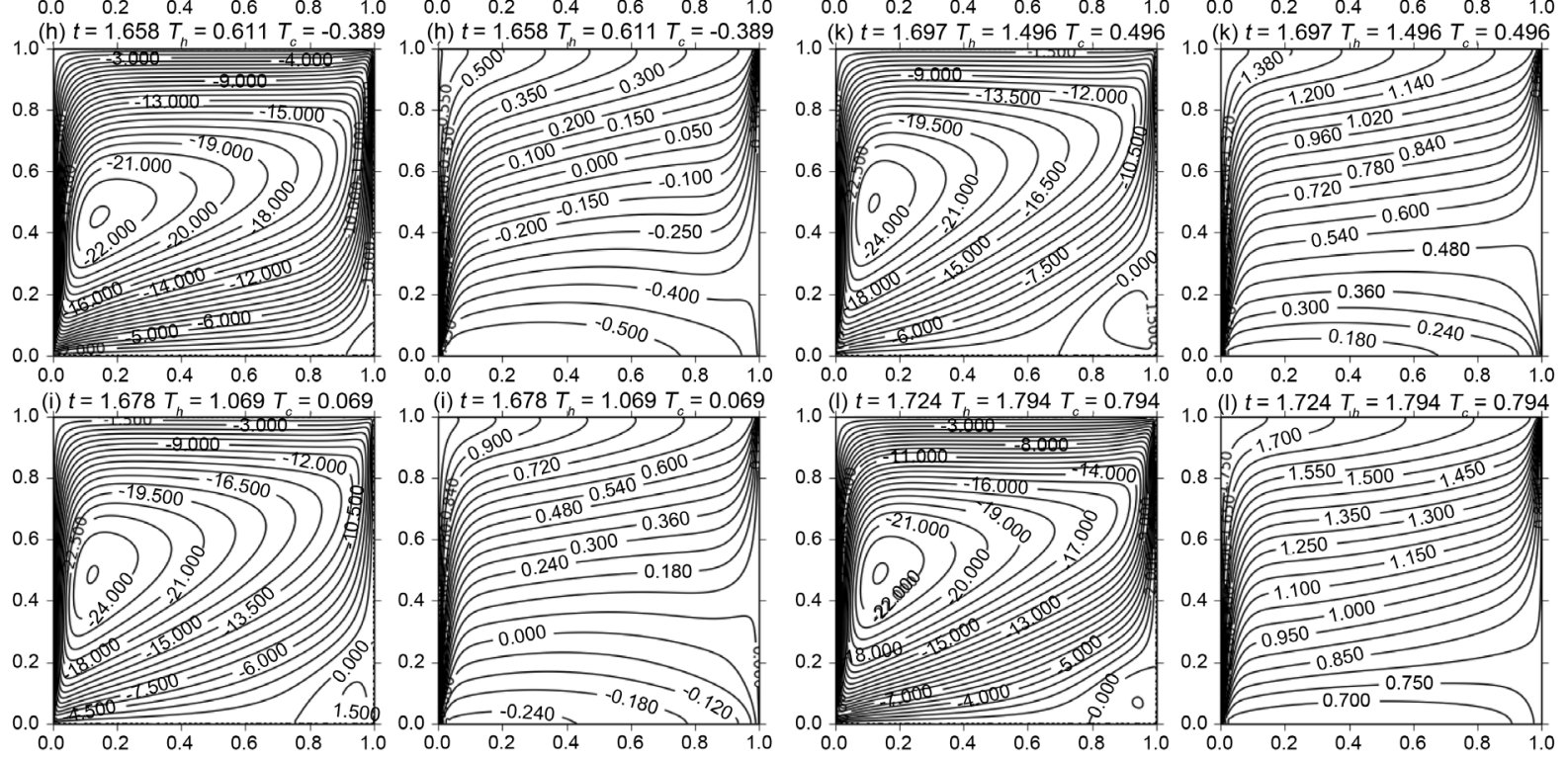

Figure 8. (a)-(l) Streamlines (left) and isotherms (right) over cycle for $R a=10^{3}, a=0.8$ and $\omega=30$. 
heat to the outside environment. The evolution of temperatures in phase opposition is the best way to remove the heat to the outside environment. When oscillatory regime is imposed, important differences are noted in terms of flow structure and of transfers heat unlike the case of constant heating; depending on the purpose, these differences can be exploited by the modeller.

\section{Acknowledgements}

We thank the Editor and the referee for their comments.

\section{References}

[1] Alves, T.A. and Altemani, C.A.C. (2012) An Invariant Descriptor for Heaters Temperature Prediction in Conjugate Cooling. International Journal of Thermal Sciences, 58, 92-101. https://doi.org/10.1016/j.ijthermalsci.2012.03.007

[2] Kuznetsov, G.V. and Sheremet, M.A. (2008) New Approach to the Mathematical Modeling of Thermal Regimes for Electronic Equipment. Russian Microelectronics, 37, 131-138. https://doi.org/10.1134/S1063739708020078

[3] Sheremet, M.A. and Pop, I. (2014) Natural Convection in a Square Porous Cavity with Sinusoidal Temperature Distributions on Both Side Walls Filled with a Nanofluid: Buongiorno's Mathematical Model. Transport in Porous Media, 105, 411-429. https://doi.org/10.1007/s11242-014-0375-7

[4] Bagchi, A. and Kulacki, F.A. (2013) Natural Convection in Superposed Fluid-Porous Layers. Springer-Verlag, New York, 80.

[5] Nield, D.A. and Bejan, A. (2006) Convection in Porous Media. 3rd Edition, Springer-Verlag, New York, 640.

[6] Pop, I. and Ingham, D.B. (2001) Convective Heat Transfer: Mathematical and Computational Modelling of Viscous Fluids and Porous Media. Elsevier, Amsterdam, 668.

[7] Vafai, K. (2005) Handbook of Porous Media. 2nd Edition, CRC Press, New York, 784.

[8] Ingham, D.B. and Pop, I. (2005) Transport Phenomena in Porous Media III. Elsevier, Oxford, 450.

[9] Baytas, A.C. and Pop, I. (2002) Free Convection in a Square Porous Cavity Using a Thermal Nonequilibrium Model. International Journal of Thermal Sciences, 41, 861-870. https://doi.org/10.1016/S1290-0729(02)01379-0

[10] Schaladow, S.G., Patterson, J.C. and Street, R.L. (1989) Transient Flow in a SideHeated Cavity at High Rayleigh Number: A Numerical Study. Journal of Fluid Mechanics, 200, 121-148. https://doi.org/10.1017/S0022112089000595

[11] Kazmierczak, M. and Chinoda, Z. (1992) Buoyancy-Driven Flow in an Enclosure with Time Periodic Boundary Conditions. International Journal of Heat and Mass Transfer, 35, 1507-1518. https://doi.org/10.1016/0017-9310(92)90040-Y

[12] Lage, J.L. and Bejan, A. (1993) The Resonance of Natural Convection in an Enclosure Heated Periodically from the Side. International Journal of Heat and Mass Transfer, 36, 2027-2038. https://doi.org/10.1016/S0017-9310(05)80134-6

[13] Lage, J.L., Junqueira, S.L.M., De Lai, F.C. and Franco, A.T. (2016) Aspect Ratio Effect on the Prediction of Boundary Layer Interference in Steady Natural Convection inside Heterogeneous Enclosures. International Journal of Heat and Mass Transfer, 92, 940-947. https://doi.org/10.1016/j.ijheatmasstransfer.2015.09.062 
[14] Seyf, H.R. and Rassoulinejad-Mousavi, S.M. (2011) An Analytical Study for Fluid Flow in Porous Media Imbedded inside a Channel with Moving or Stationary Walls Subjected to Injection/Suction. Journal of Fluids Engineering, 133, 9.

[15] Rassoulinejad-Mousavi, S.M. and Yaghoobi, H. (2014) Effect of Non-Linear Drag Term on Viscous Dissipation in a Fluid Saturated Porous Medium Channel with Various Boundary Conditions at Walls. Arabian Journal for Science and Engineering, 39, 1231-1240. https://doi.org/10.1007/s13369-013-0676-0

[16] Abourida, B., Hasnaoui, M. and Douamna, S. (1998) Natural Convection in a Square Cavity with Vertical Boundaries Submitted to Periodic Temperatures. Revue Générale de Thermique, 37, 788-800. https://doi.org/10.1016/S0035-3159(98)80005-6

[17] Saeid, N.H. (2005) Natural Convection in a Square Porous Cavity with an Oscillating Wall Temperature. The Arabian Journal for Science and Engineering, 31, 35-46.

[18] Malomar, G.E.B., Gueye, A., Mbow, C., Traore, V.B. and Beye, A.C. (2016) Numerical Study of Natural Convection in a Square Porous Cavity Thermally Modulated on Both Side Walls. American Journal of Engineering and Applied Sciences, 9, 591 598. https://doi.org/10.3844/ajeassp.2016.591.598

[19] Walker, K.L. and Homsy, G.M. (1978) Convection in a Porous Cavity. Journal of Fluid Mechanics, 87, 449-474. https://doi.org/10.1017/S0022112078001718

[20] Bejan, A. (1979) On the Boundary Layer Regime in a Vertical Enclosure Filled with a Porous Medium. Letters in Heat and Mass Transfer, 6, 93-102. https://doi.org/10.1016/0094-4548(79)90001-8

[21] Beckermann, C., Viskanta, R. and Ramadhyani, S. (1986) A Numerical Study of Non-Darcian Natural Convection in a Vertical Enclosure Filled with a Porous Medium. Numerical Heat Transfer Applications, 10, 557-570.

[22] Moya, S.L., Ramos, E. and Sen, M. (1987) Numerical Study of Natural Convection in a Tilted Rectangular Porous Material. International Journal of Heat and Mass Transfer, 30, 741-756. https://doi.org/10.1016/0017-9310(87)90204-3

[23] Manole, D.M. and Lage, J.L. (1992) Numerical Benchmark Results for Natural Convection in a Porous Medium Cavity. Heat and Mass Transfer in Porous Media, Anaheim, 8-13 November 1992, 55-60. 


\section{Nomenclature}

$a$, Dimensionless amplitude

$g$, Gravity intensity, $\mathrm{m} \cdot \mathrm{s}^{-2}$

$k$, Permeability of the porous medium, $\mathrm{m}^{2}$

$t$, Dimensionless time

$T$, Dimensionless temperature

$(x, y)$, Dimensionless coordinates

$R a$, Rayleigh number for porous medium

$\Delta T^{\prime}$, Average temperature difference, $\mathrm{K}$

$\overline{N u c}$, Average Nusselt number at the cold wall

$\overline{N u h}$, Average Nusselt number at the hot wall

\section{Greek Symbols}

$\alpha$, Effective thermal diffusivity, $\mathrm{m}^{2} \cdot \mathrm{s}^{-1}$

$\beta_{T}$, Coefficient of thermal expansion, $\mathrm{K}^{-1}$

$\mu$, Dynamic viscosity, $\mathrm{kg} \cdot \mathrm{m}^{-1} \cdot \mathrm{s}^{-1}$

$\phi$, Dimensionless phase angle

$\sigma$, Ratio of heat capacity of porous medium to that of fluid

$\omega$, Dimensionless frequency

$\tau$, Dimensionless period

$\psi$, Dimensionless stream function

$\rho_{0}$, Reference density of fluid, $\mathrm{kg} \cdot \mathrm{m}^{-3}$

\section{Superscript}

('), Dimensional variables

(-), Average values

\section{Subscript}

c, Cold

h, Hot

f, Fluid

min, Minimum value

max, Maximum value 
Submit or recommend next manuscript to SCIRP and we will provide best service for you:

Accepting pre-submission inquiries through Email, Facebook, LinkedIn, Twitter, etc. A wide selection of journals (inclusive of 9 subjects, more than 200 journals)

Providing 24-hour high-quality service

User-friendly online submission system

Fair and swift peer-review system

Efficient typesetting and proofreading procedure

Display of the result of downloads and visits, as well as the number of cited articles Maximum dissemination of your research work

Submit your manuscript at: http://papersubmission.scirp.org/

Or contact ojfd@scirp.org 